\title{
Primary orbital melanoma
}

\section{Vivian WK Hui, TC Lau, Lawrence PW Ng, Hunter KL Yuen, W Cheuk *}

Hong Kong Med J 2021;27:223.e1-2

https://doi.org/10.12809/hkmj208788

A 68-year-old Chinese man presented with proptosis, epiphora and discomfort in the right eye associated with impaired visual acuity. Contrast computed tomography scan revealed a $3.3 \mathrm{~cm} \times 2.1 \mathrm{~cm}$ heterogeneously enhancing mass at the superotemporal aspect of the right orbit with displacement of the lateral rectus muscle (Fig 1). A pigmented tumour with dense adhesions to the surrounding structures was found in orbitotomy with leakage of the pigmented content upon surgical exploration. The lesion was excised as much as possible. Detailed clinical examination showed no evidence of intraocular melanoma, conjunctival or eyelid melanoma, or melanoma anywhere else. Systemic examination and whole-body positron emission tomography-computed tomography scan were unremarkable. The patient was alive with no evidence of disease at 9-month follow-up.

The tumour was a solid, dark brownish multinodular mass covered by fat and skeletal muscle, comprising lobulated sheets of epithelioid and spindly melanocytes with vesicular chromatin, prominent nucleoli, and variable amounts of melanin pigment (Figs 2 to 4). The mitotic count was 1 per $10 \mathrm{HPFs}$. The neoplastic melanocytes were positive for S100 and SOX10 but negative for BRAF. BRCA1-associated protein 1 immunostaining was intact. Sanger sequencing revealed a GNA11 Q209L mutation. No mutation was found in GNAQ, NRAS, KRAS, BRAF or KIT.

Ocular melanoma most frequently involves the uveal tract (choroid, ciliary body, and iris) and the conjunctiva, where melanocytes are normally

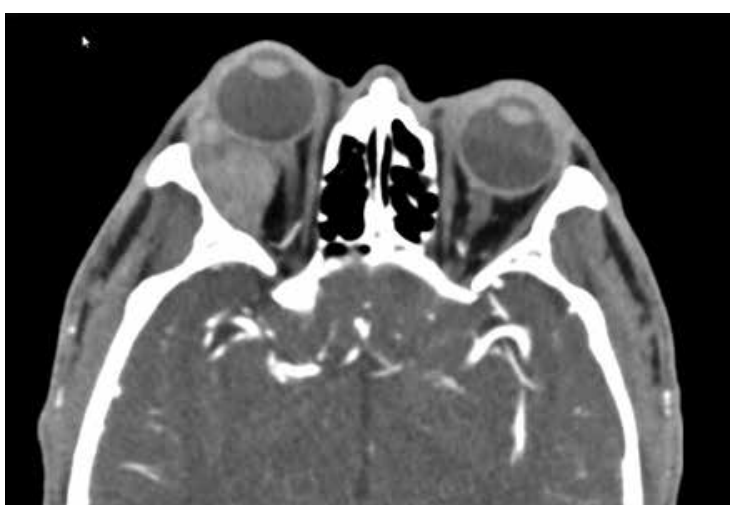

FIG I. Contrast computed tomography scan showing a contrast-enhancing tumour behind the right globe displacing the optic nerve

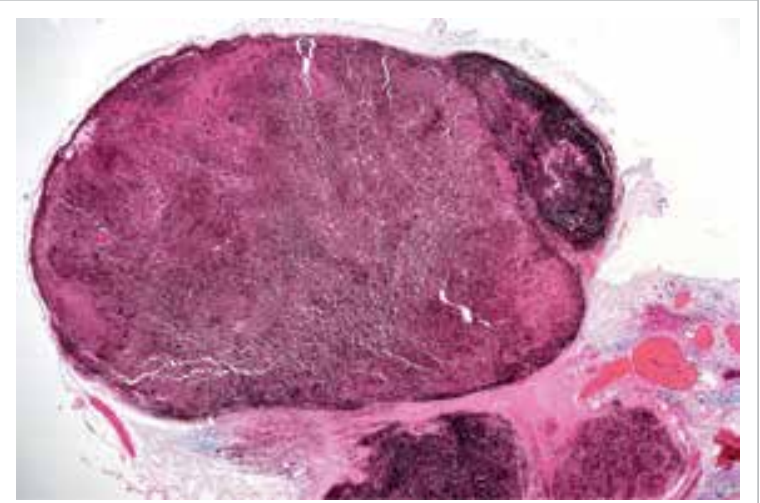

FIG 2. Micrograph showing multinodular mass with variable amount of melanin pigment in different areas $(\times 40)$

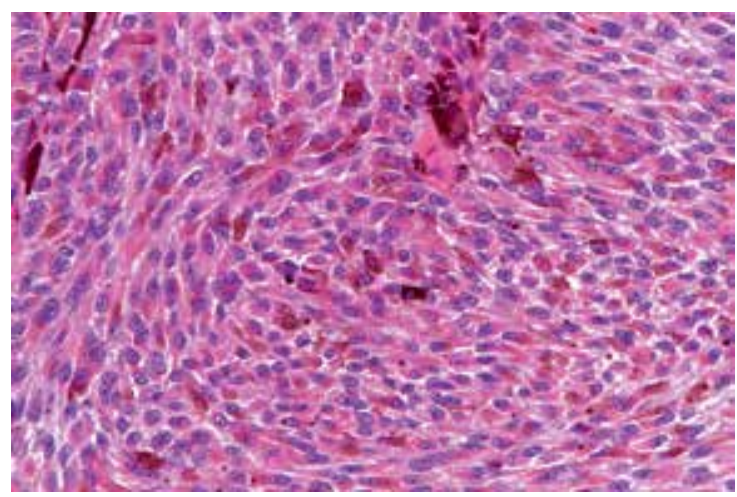

FIG 3. Large sheets of epithelioid (left upper field) and spindly (right lower field) melanoma cells with pale chromatin and prominent nucleoli were found. There were scattered histiocytes with abundant cytoplasmic melanin pigment $(\times 200)$

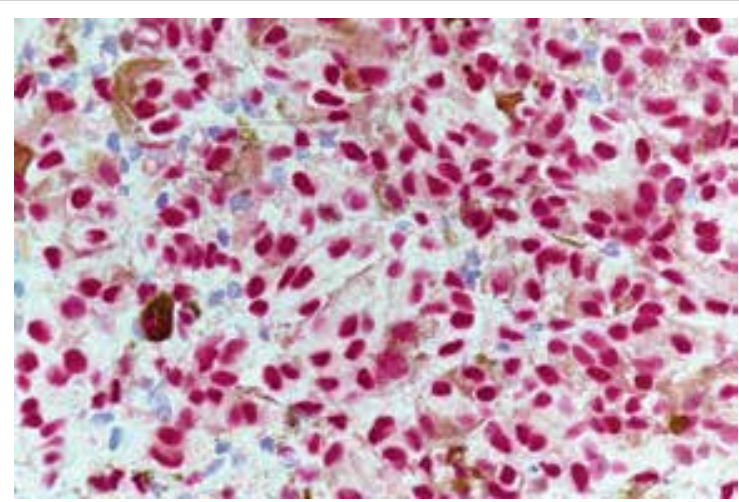

FIG 4. Melanoma cells were positive on SOXIO (red chromogen) immunohistochemical staining $(\times 200)$ 
present. According to The Cancer Genome Atlas uveal melanoma project, mutually exclusive mutations in GNAQ, GNA11, CYSLTR2 and PLCB4 are the tumour-initiating mutations whereas $B A P 1$, EIF1AX and SF3B1/SRSF2 mutations are associated with prognosis in uveal melanoma. ${ }^{1}$ In contrast, conjunctival melanoma typically exhibits $B R A F$, $N R A S$ and NF1 mutations of the MAPK pathway with UV-induced $\mathrm{C} \rightarrow \mathrm{T}$ mutation signature. ${ }^{2}$ Orbital soft tissue melanoma is uncommon with $>90 \%$ representing secondary tumours as a result of contiguous spread or metastasis from uveal, conjunctival, cutaneous or sinonasal melanomas. ${ }^{3}$ Primary orbital melanoma is exceedingly rare and has been postulated to arise in embryological remnants of melanocytes arrested in the region since a substantial proportion of cases are associated with blue nevus, orbital melanocytosis or oculodermal melanocytosis (nevus of Ota). Alternatively, it may arise from isolated melanocytes found in the optic nerve sheath, orbital fat, extraocular muscles or orbital periosteum. The majority of patients are white Europeans in their fourth to fifth decade. Histologically, it comprises epithelioid, spindle or mixed populations of neoplastic melanocytes that are frequently pigmented but with a lesser degree of nuclear pleomorphism than their cutaneous and mucosal counterparts. The prognosis is generally poor but a small proportion of patients survive long term. The most frequent site of metastasis is the liver. ${ }^{3,4}$ The clinicopathological features of primary orbital melanoma are very similar to those of uveal melanoma. The findings of GNAQ, SF3B1, EIF1AX mutations ${ }^{5}$ and GNA11 mutation in this case provide further evidence that primary orbital melanoma has a close pathogenic relationship with uveal melanoma.

\section{Author contributions}

All authors contributed to the concept or design of the study, acquisition of the data, analysis or interpretation of the data, drafting of the manuscript, and critical revision of the manuscript for important intellectual content. All authors had full access to the data, contributed to the study, approved the final version for publication, and take responsibility for its accuracy and integrity.

\section{Conflicts of interest}

As an adviser of the journal, HKL Yuen was not involved in the peer review process. Other authors have disclosed no conflicts of interest.

\section{Declaration}

This case was presented as a poster at the Annual Scientific Meeting of The College of Ophthalmologists of Hong Kong and Hong Kong Ophthalmological Society on 14 to 15 December 2019.

\section{Funding/support}

This pictorial medicine paper received no specific grant from any funding agency in the public, commercial, or not-forprofit sectors.

\section{Ethics approval}

The patient was treated in accordance with the tenets of the Declaration of Helsinki. Informed consent was obtained from the patient.

${ }^{1}$ VWK Hui, MB, ChB

2 TC Lau, PhD

2 LPW Ng, MSc

HKL Yuen, FRCOphth

${ }^{2}$ W Cheuk *, FHKCPath

${ }^{1}$ Department of Ophthalmology, Hong Kong Eye Hospital, Hong Kong

Department of Pathology, Queen Elizabeth Hospital, Hong Kong

* Corresponding author: cwzz01@ha.org.hk

\section{References}

1. Bakhoum MF, Esmaeli B. Molecular characteristics of uveal melanoma: insights from the Cancer Genome Atlas (TCGA) Project. Cancers (Basel) 2019;11:1061.

2. Swaminathan SS, Field MG, Sant D, et al. molecular characteristics of conjunctival melanoma using wholeexome sequencing. JAMA Ophthalmol 2017;135:1434-7.

3. Rose AM, Luthert PJ, Jayasena CN, Verity DH, Rose GE. Primary orbital melanoma: presentation, treatment, and long-term outcomes for 13 patients. Front Oncol 2017;7:316.

4. Shields JA, Shields CL. Orbital malignant melanoma: the 2002 Sean B Murphy lecture. Ophthalmic Plast Reconstr Surg 2003;19:262-9.

5. Rose AM, Luo R, Radia UK, et al. Detection of mutations in SF3B1, EIF1AX and GNAQ in primary orbital melanoma by candidate gene analysis. BMC Cancer 2018;18:1262. 